\title{
ESPACES HOMOGENES DE STEIN DES GROUPES DE LIE COMPLEXES, II
}

\author{
YOZÔ MATSUSHIMA
}

Ce travail est une suite du mémoire [8] paru dans ce journal. Dans [8], on a étudié la structure du groupe connexe d'isotropie d'un espace homogène de Stein $G / H$ d'un groupe de Lie semi-simple complexe et connexe $G$ et on a montré que le groupe $H$ est égal au complexifié d'un sous-groupe compact maximal de $H$ ([8], Théorème 3 ). Le but essentiel de ce travail est d'étendre ce résultat au cas d'un espace homogène de Stein d'un groupe de Lie complexe et connexe quelconque.

Soit $G / H$ un espace homogène de Stein d'un groupe de Lie complexe et connexe $G$. Supposons que $H$ soit connexe et que $G$ opère de manière presque effective sur $G / H$. D'après un résultat de [7], $G$ est alors un groupe de Stein. Soient $K$ et $L$ des sous-groupes compacts maximaux de $G$ et $H$ respectivement tels que $K \supset L$ et soient $\widetilde{K}$ et $\widetilde{L}$ leurs complexifiés. On démontre que $\widetilde{L}$ est égal à la composante connexe de l'élement neutre du groupe $\widetilde{K} \cap H$ (Théorème 2). Pour établir ce théorème on démontre d'abord que, si un groupe de Lie complexe opère sur une variété complexe et connexe de manière holomorphe et presque effective, le groupe connexe d'isotropie satisfait à une certaine condition remarquable (Théorème 1 ).

Si le groupe $G$ est égal au complexifié d'un sous-groupe compact maximal, la condition pour le groupe $H$ trouvée dans le théorème 2 est suffisante pour que $G / H$ soit une variété de Stein, comme on le voit du théorème 5 de [7]. On démontre ici que, si $G$ est un groupe de Stein nilpotent, cette condition est suffisante pour que $G / H$ soit une variété de Stein.

Dans le paragraphe 4, on étabit un théorème de décomposition de la variété d'un groupe de Lie complexe et connexe, ce qui est analogue à un théorème trouvé par Mostow [9] dans le cas d'un groupe de Lie réel. On retrouve ainsi le théorème 1 de [8] dans une forme plus précise.

Received Novenber 29, 1960. 
1. Dans tout ce qui suit, on désignera par $\mathfrak{g}, \mathfrak{h}, \mathfrak{l}$ etc. les algèbres de Lie des groupes de Lie $G, H, L$ etc. Soit $G$ un groupe de Lie complexe et connexe et soit $K$ un sous-groupe compact maximal de $G$. On dit que $G$ est un groupe de Stein, si $\mathfrak{k} \cap i \cdot \mathfrak{k}=(0)$. D'après [7], Théorème 1 , la variété sous-jacente d'un groupe de Stein est une variété de Stein. Soit $G$ un groupe de Stein et soit $K$ un sous-groupe compact maximal de $G$. Soit $\widetilde{f}$ la sous-algèbre complexe de $g$ engendrée par la sous-algèbre réelle $\mathfrak{f}: \widetilde{\mathfrak{f}}=\mathfrak{f}+i \cdot \mathfrak{k}$. L'algèbre $\widetilde{\mathfrak{f}}$ est isomorphe à la complexification $\mathfrak{t}^{c}=\mathfrak{l} \otimes C$ de $\mathfrak{f}, C$ désignant le corps des nombres complexes. Le sous-groupe de Lie complexe et connexe de $G$ correspondant à $\widetilde{f}$ sera désigné par $\tilde{K}$ et appelé le complexifié de $K$ dans $G$. Le groupe $\tilde{K}$ est fermé dans $G$ [8].

Definition. Soit $G$ un groupe de Lie complexe et connexe et soit $K$ un sous-groupe compact maximal de $G$. On dit que le groupe $G$ satisfait à la condition $(Q)$ si la suivante soit vérifiée:

$(Q)$. Le groupe $G$ est de Stein et il existe un sous-groupe de Lie fermé, invariant et complexe $N$ de $G$ qui est résoluble, connexe et simplement, connexe tel que $G=\tilde{K} \cdot N, \tilde{K} \cap N=(e)$.

Un théorème de Hochschild-Mostow ([6], Theorem 4.2) peut être énoncé sous la forme suivante.

Proposition 1.1 (Hochschild-Mostow). Soit $G$ un groupe de Lie complexe et connexe. Si $G$ possède une représentation linéaire holomorphe fidèle, alors $G$ satisfait à la condition $(Q)$.

La réciproque de cette proposition est aussi vraie, comme il a été démontré par Hochschild et Mostow ([6], Theorem 3.6). Mais on ne l'utilisera pas dans la suite.

2. THÉORÈME 1. Soit G un groupe de Lie complexe opérant sur une variété complexe et connexe $V$ de manière holomorphe et presque effective. Alors le groupe connexe d'isotropie de $G$ en un point quelconque de $V$ satisfait à la condition $(Q)$.

Etablissons d'abord quelques lemmes.

LeMme 2.1. Soit $G$ un groupe de Lie complexe et connexe et soit $M$ un sous-groupe de Lie fermé, invariant et complexe de $G$ qui est résoluble, connexe 
et simplement connexe. Si le groupe quotient $G / M$ satsfait à la condition $(Q)$, il en est de même de G.

Soit $G^{\prime}=G / M$ et soit $\pi$ l'homomorphisme canonique de $G$ sur $G^{\prime}$. Soit $K^{\prime}$ un sous-groupe compact maximal de $G^{\prime}$. Le groupe $M$ étant connexe, il existe un sous-groupe compact maximal $K$ de $G$ tel que $\pi \cdot K=K^{\prime}$ (cf. [j]). Puisque $M$ est résoluble et simplement connexe, tout sous-groupe compact maximal de $M$ se réduit à $(e)$. Par suite, $K \cap M=(e)$ et la restriction de $\pi$ à $K$ est une bijection de $K$ sur $K^{\prime}$. On va montrer d'abord que $G$ est un groupe de Stein.

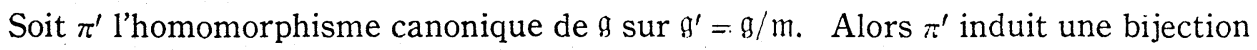
de $\mathfrak{f}^{*}$ sur $\mathfrak{*}^{\prime}$. Soit $X \in \mathfrak{f} \cap i$ f. Alors $\pi^{\prime}(X) \in \mathfrak{l}^{\prime} \cap i^{\prime \prime}$. Puisque $G^{\prime}$ satisfait à la condition $(Q), G^{\prime}$ est de Stein et done $\sharp^{\prime} \cap i^{\prime \prime}=(0)$. Par suite, $\pi^{\prime}(X)=0$ et, l'application $\pi^{\prime}$ étant bijective dans $\mathfrak{f}$, on a $X=0$. Alors $\mathfrak{f} \cap i \mathfrak{f}=(0)$ et $G$ est de Stein. Puisque $\pi^{\prime}(\widetilde{f})=\widetilde{f}^{\prime}$, l'homomorphisme $\pi$ induit un homomorphisme $\pi_{1}$ de $\widetilde{K}$ sur $\widetilde{K}^{\prime}$. Puisque $\widetilde{K}$ et $\widetilde{K}^{\prime}$ sont les complexifiés de leurs sous-groupes compacts maximaux $K$ et $K^{\prime}$ et puisque $\pi_{1}$ induit une bijection de $K$ sur $K^{\prime}$, on voit que $\pi_{1}$ est bijectif (voir [8], l'appendice).

Cela posé, d'après l'hypothèse du lemme, il existe un sous-groupe de Lie fermé, invariant et complexe $N^{\prime}$ de $G^{\prime}$ qui est résoluble, connexe et simplement connexe tel que $G^{\prime}=\widetilde{K^{\prime}} \cdot N^{\prime}, \widetilde{K^{\prime}} \cap N^{\prime}=(e)$. Soit $N$ la composante connexe de l'élément neutre de $\pi^{-1}\left(N^{\prime}\right)$. Alors $N$ est un sous-groupe de Lie fermé, invariant et complexe de $G$ contenant $M$ et $\pi \cdot N=N / M=N^{\prime}$. Les groupes $M$ et $N^{\prime}$ étant résoluble et simplement connexe, le groupe $N$ l'est aussi. On va montrer que $G=\widetilde{K} . N, \tilde{K} \cap N=(e)$. Puisque $\widetilde{K^{\prime}}=\widetilde{K} \cdot M / M, N^{\prime}=N / M$ et $G^{\prime}=\widetilde{K^{\prime}} \cdot N^{\prime}$, on voit que $G=\widetilde{K} . N$. Désignons par $\alpha$ et $\theta$ respectivement les homomorphismes canoniques de $G$ sur $G / N$ et de $G^{\prime}=G / M$ sur $G / N$. Alors $\alpha=\theta \circ \pi$. Soit maintenant $x \in \tilde{K} \cap N$. Le noyau de $\alpha$ étant egal à $N$, on a $\alpha(x)=e$ et par suite $\theta(\pi(x))$ $=e$. Le noyau de $\theta$ étant égal à $N^{\prime}$ on a $\pi(x) \in N^{\prime}$. D'autre part, $x \in \widetilde{K}$ et donc $\pi(x) \in \widetilde{K^{\prime}}$. Alors $\pi(x)=e$, car $\widetilde{K}^{\prime} \cap N^{\prime}=(e)$. Or, on a déjà démontré que la restriction $\pi_{1}$ de $\pi$ à $\widetilde{K}$ est une bijection de $\widetilde{K}$ sur $\widetilde{K^{\prime}}$. Par conséquent, on a $x=e$ et $\tilde{K} \cap N=(e)$. Le groupe $G$ satisfait donc à la condition $(Q)$.

Lemme 2.2. Soit $G$ un groupe de Lie complexe et connexe et soit $D$ un sous-groupe invariant discret de $G$. Si le group quotient $G / D$ satisfait à la condition $(Q)$, le groupe $G$ satisfait aussi à la même condition. 
Soit $G^{\prime}=G / D$ et soit $\pi$ (resp. $\pi^{\prime}$ ) l'homomorphisme canonique de $G$ (resp. de g) sur $G^{\prime}$ (resp. sur $g^{\prime}$ ). Alors $\pi^{\prime}$ est une bijection. Soit $K$ un sous-groupe compact maximal de $G$ et soit $K^{\prime}$ un sous-groupe compact maximal de $G^{\prime}$ contenant $\pi . K$. Puisque le groupe $G^{\prime}$ satisfait à la condition $(Q)$, il existe un sousgroupe de Lie fermé, invariant et complexe $N^{\prime}$ de $G^{\prime}$ qui est résoluble, connexe et simplement connexe tel que $G^{\prime}=\widetilde{K}^{\prime} \cdot N^{\prime}, \widetilde{K}^{\prime} \cap N^{\prime}=(e)$. Soit $R^{\prime}$ le radical de $G^{\prime}$. Alors $R^{\prime}$ contient $N^{\prime}$ et le groupe quotient $R^{\prime} / N^{\prime}$ est égal au centre connexe de $G^{\prime} / N^{\prime}$. Soit $R$ le radical de $G$. On a alors $\pi \cdot R=R^{\prime}$. Soit maintentant $N$ la composante connexe de l'élément neutre de $\pi^{-1}\left(N^{\prime}\right)$. Alors $N$ est contenu dans $R$ et $N$ est un revêtement de $N^{\prime}$. Puisque $N^{\prime}$ est simplement connexe, l'homomorphisme $\pi$ induit une bijection de $N$ sur $N^{\prime}$. Par suite, $N$ est simplement connexe et résoluble. On va montrer que $G / N$ est de Stein. Le groupe quotient $G^{\prime} / N^{\prime}$ étant isomorphe à $\widetilde{K^{\prime}}, G^{\prime} / N^{\prime}$ est de Stein. D'après [7], Proposition 6, on

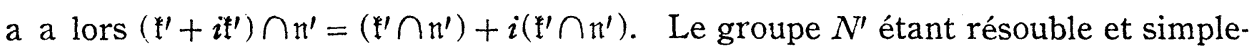
ment connexe, tout sous-groupe compact maximal de $N^{\prime \prime}$ se réduit à $(e)$ et par suite $\mathfrak{l}^{\prime} \cap \mathfrak{n}^{\prime}=(0)$. Alors $\left(\mathfrak{q}^{\prime}+i \mathfrak{l}^{\prime}\right) \cap \mathfrak{n}^{\prime}=(0)$. Or, $\pi^{\prime}$ est bijectif et $\pi^{\prime}(\mathfrak{f}) \subset \mathfrak{q}^{\prime}$ et $\pi^{\prime}(\mathfrak{n})=\mathfrak{n}^{\prime}$. Par conséquent, on a $(\mathfrak{f}+\mathfrak{i}) \cap \mathfrak{n}=(0)$. Alors $G / N$ est de Stein d'après [7], Proposition 6. Le groupe $G / N$ étant de Stein, le sous-groupe $R / N$ est aussi de Stein. D'autre part, utilisant le fait que $R^{\prime} / N^{\prime}$ est le centre connexe de $G^{\prime} / N^{\prime}$, on voit que $R / N$ est le centre connexe de $G / N$. On sait que un groupe de Stein abélien est isomorphe au gronpe $C^{n} \times C^{* m}(m, n \geqq 0)$ ([7], Proposition 2). Il existe alors des sous-groupes de Lie fermés, invariants, complexes et connexes $M$ et $L$ de $R$ contenant $N$ tels que $R / N=(M / N) \times(L / N), M / N \cong C^{n}, L / N \cong C^{* m}$. Le groupe $R / N$ étant le centre connexe de $G / N$, le groupe $M$ est invariant dans $G$. De plus, les groupes $N$ et $M / N$ étant résolubles et simplement connexes, il en est de même de $M$. On va montrer que le groupe $G / M$ est de Stein. En effet, le groupe $G / R$ étant semi-simple, $G / R$ est de Stein. D'après [7], Proposition 6 , on a alors $(\mathfrak{f}+i \mathfrak{f}) \cap \mathfrak{r}=(\mathfrak{n} \cap \mathfrak{r})+i(\mathfrak{r} \cap \mathfrak{r})$. $\mathfrak{f} \cap \mathfrak{r}$ est l'algèbre de Lie du sous-groupe compact maximal $K \cap R$ de $R$. D'autre part, $R / M$ étant isomorphe à $L / N, R / M$ est de Stein. Par suite, $((\sharp \cap \mathfrak{r})+i(\sharp \cap \mathfrak{r})) \cap m=(\sharp \cap m)+i(\sharp \cap m)$.

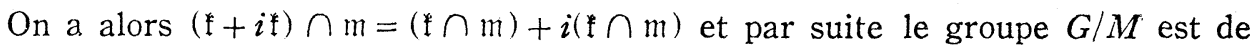
Stein. On va montrer maintenant que $G / M$ est égal au complexifié du sousgroupe compact maximal $K . M / M$. En effet, le groupe $G / N$ est réductif et de Stein et le centre connexe de $G / N$ est égal à $R / N$. Le complexifié $\widetilde{K} . N / N$ du sous-groupe compact maximal $K . N / N$ de $G / N$ coïncide alors avec le produit de 
$L / N$ et de la partie semi-simple de $G / N$. Par suite, on a $G / N=(\widetilde{K} . N / N) .(M / N)$. Alors $G=\widetilde{K} . M$ et donc $G / M=\widetilde{K} . M / M$. On a ainsi montré que $G / M$ est de Stein et que $G / M$ est égal au complexifié d'un sous-groupe compact maximal. Alors $G / M$ satisfait à la condition $(Q)$. Le groupe $M$ étant résoluble et simplement connexe, $G$ satisfait à la condition $(Q)$ d'après le lemme 2.1.

Lеммe 2.3. Soit $H$ un groupe de Lie complexe et connexe opérant de maniere holomorphe et presque effective sur une variété complexe et connexe $V$. Soit a un point de $V$ tel que $h(a)=a$ quel que soit $h \in H$. Soit $\rho$ l'homomorphisme canonique de $H$ dans le groupe linéaire d'isotropie de $H$ en le point a. Alors la composante connexe de l'élément neutre du noyau de o est résoluble et simplement connexe ${ }^{11}$.

Soit $L_{n}^{r}$ le groupe de $r$-jets "holomorphes" inversibles de source et but $a$, où $n$ désigne la dimension complexe de $V$ (pour la notion de jets, voir Ehresmann [3]). Le groupe $L_{n}^{r}$ est un groupe de Lie complexe et connexe et on peut identifier $L_{n}^{1}$ avec $G L(n, C)$. Soit $\pi_{s}^{r}(r>s)$ l'homomorphisme canonique de $L_{n}^{r}$ sur $L_{n}^{s}$ défini par $\pi_{s}^{r}\left(j_{a}^{r} f\right)=j_{a}^{s} f$. Le noyau de $\pi_{1}^{r}$ est résoluble, connexe et simplement connexe. Soit maintenant $\rho_{r}$ l'homomorphisme de $H$ dans $L_{n}^{r}$ défini par $\rho_{r}(h)=j_{a}^{r} h, h \in H$. Alors $\rho=\rho_{1}$ et $\rho_{s}=\pi_{s}^{r} \circ \rho_{r}(r>s)$. Soit $N_{r}$ la composante connexe de l'élément neutre du noyau de $\rho_{r}$. On a alors $N_{1} \supset N_{2} \supset \cdots$ Il existe alors un entier $r>0$ tel que $N_{r}=N_{r+1}=\cdots$ Soit $h \in N_{r}$. Alors $j_{a}^{s} h=e$ quel que soit l'entier positif $s$. On voit alors que la transformation $h$ de $V$ laisse fixe tout point dans un voisinage suffisamment petit du point $a$. La variété $V$ étant connexe, la transformation $h$ de $V$ est alors la transformation identique. Or, on a supposé que $H$ opère sur $V$ de manière presque effective. Alors le groupe $N_{r}$ est discret et donc se réduit à $(e)$. Par conséquent, $\rho_{r}$ est un isomorphisme local. D'autre part, puisque $\rho_{1}=\pi_{1}^{r} \circ \rho_{r}$, le groupe $\rho_{r}\left(N_{1}\right)$ est dans le noyau $S_{r}$ de l'homomorphisme $\pi_{1}^{r}$ de $L_{n}^{r}$ sur $L_{n}^{1}$. Le groupe $S_{r}$ étant résoluble, connexe et simplement connexe, le sous-groupe de Lie connexe $\rho_{r}\left(N_{1}\right)$ de $S_{r}$ est aussi résoluble et simplement connexe [2]. D'après ce que l'on a déjà montré, le groupe $N_{1}$ est un revêtement de $\rho_{r}\left(N_{1}\right)$. Par suite, $N_{1}$ est résoluble et simplement connexe.

Démonstration du théorème 1 . Soit $a$ un point de $V$ et soit $H$ le groupe

1) cf. Grauert [4]. 
connexe d'isotropie de $G$ en le point $a$. Soit $\rho$ l'homomorphisme canonique de $H$ dans le groupe linéaire d'isotropie de $H$ en le point $a$. Soit $N$ le noyau de $\rho$ et soit $N_{0}$ la composante connexe de l'élément neutre de $N$. L'homomorphisme $\rho$ induit une représentation linéaire holomorphe fidèle du groupe quotient $H / N$. D'après la proposition 1.1. le groupe $H / N$ satisfait à la condition $(Q)$. Le groupe $H / N$ étant isomorphe au quotient de $H / N_{0}$ par le sous-groupe invariant diecret $N / N_{0}$, le groupe $H / N_{0}$ satisfait à la condition $(Q)$ d'après le lemme 2.2. D'après le lemme 2.3, le groupe $N_{0}$ est résoluble et simplement connexe. Alors, d'après le lemme 2.1, le groupe $H$ satisfait à la condition $(Q)$. Le théorème 1 est ainsi démontré.

Remarque. Un exemple facile montre que l'homomorphisme de $H$ dans le groupe linéaire d'isotropie n'est pas toujours injectif.

3. THÉORÈme 2. Soit $G$ un groupe de Lie complexe et connexe et soit $H$ un sous-groupe de Lie fermé, complexe et connexe de $G$. Soient $K$ et $L$ respectivement des sous-groupes compacts maximaux de $G$ et $H$ tels que $K \supset L$. Supposons que $G / H$ soit une variété de Stein et que $G$ opère de manière presque effective sur $G / H$. Alors le groupe $\widetilde{L}$ est égal à la composante connexe de l'élément neutre du groupe $\tilde{K} \cap H$.

Remarque. Ce théorème généralise le théorème 3 de [7]. En effet, si $G$ est semi-simple, on a $\widetilde{K}=G$ et donc $\tilde{K} \cap H=H=\widetilde{L}$.

Etablissons d'abord quelques lemmes.

Lemme 3.1. Soit $R$ un groupe de Lie complexe et connexe et soit $R_{1}$ un sous-groupe de Lie fermé, complexe et connexe de $R$. Supposons que $R$ soit résoluble et simplement connexe. Il existe alors des sous-espaces vectoriels complexes $\mathrm{m}_{1}, \ldots, \mathrm{m}_{k}$ de dimension complexe 1 de l'algèbre de Lie $\mathfrak{r}$ de $R$ vérifiant les conditions suivantes:

1) $\mathfrak{r}=\mathfrak{m}_{1}+\cdots+\mathfrak{m}_{k}+\mathfrak{r}_{1}$ (somme direct);

2) tout elément $x$ de $R$ s'écrit de manière unique sous la forme $x=\exp X_{1}$ $\cdots \exp X_{k} y, y \in R_{1}, X_{i} \in \mathfrak{m}_{i}(i=1, \ldots, k)$.

Démontrons le lemme par récurrence sur la dimension complexe de $R$. Si la dimension complexe de $R$ est égale à 1 , le lemme est trivial. Supposons donc que le lemme soit démontré pour le groupe de dimension complexe plus petite 
que $n$ et soit $R$ un groupe de dimension complexe $n$. Remarquons d'abord que, le groupe $R$ étant résoluble et simplement connexe, tout sous-groupe de Lie connexe de $R$ est fermé et simplement connexe [2].

$1^{\circ}$. Supposons que $\mathfrak{r}_{1}$ contient un idéal complexe a de $\mathfrak{r}$ de dimension positive. Soit $R^{\prime}=R / A$ et $R_{1}^{\prime}=R_{1} / A$. Le groupe $R^{\prime}$ est aussi simplement connexe. Soit $\pi$ (resp. $\pi^{\prime}$ ) l'homomorphisme canonique de $R$ (resp. de r) sur $R^{\prime}$ (resp. sur $\left.\mathfrak{r}^{\prime}\right)$. D'après l'hypothèse de récurrence, il existe des sous-espaces vectoriels complexes $\mathrm{m}_{1}^{\prime}, \ldots, \mathrm{m}_{k}^{\prime}$ de diemension complexe 1 de $\mathfrak{r}^{\prime}$ vérifiant les conditions 1 ) et 2) pour les groupes $R^{\prime}$ et $R_{1}^{\prime}$. Soient $\mathrm{m}_{1}, \ldots, \mathrm{m}_{k}$ des sous-espaces vectoriels de dimension 1 de $r$ tels que $\pi^{\prime}\left(m_{i}\right)=\mathfrak{m}_{i}^{\prime}(i=1, \ldots, k)$. Il n'est pas difficile de voir que les sous-espaces $\mathfrak{m}_{1}, \ldots, \mathfrak{m}_{k}$ satisfont aux conditions 1 ) et 2 ).

$2^{\circ}$. Supposons que $\mathfrak{r}_{1}$ est une sous-algèbre complexe maximale de $r$. L'algèbre de Lie $\mathfrak{r}$ étant résoluble et complexe, il existe un idéal complexe a de dimension complexe 1 de $r$. Puisque $a+r_{1}$ est une sous-algèbre complexe contenant $\mathfrak{r}_{1}$, on a $\mathfrak{r}_{1}=\mathfrak{a}+\mathfrak{r}_{1}$ ou $\mathfrak{r}=\mathfrak{a}+\mathfrak{r}_{1}$. Si $\mathfrak{r}_{1}=\mathfrak{a}+\mathfrak{r}_{1}$, on $a \mathfrak{a} \subset \mathfrak{r}_{1}$ et c'est le cas déjà considéré. Supposons donc que $\mathrm{r}=a+r_{1}$. La dimension complexe de a étant égale à 1 , cette somme est directe et on a $R=A \cdot R_{1}$. On va montrer que $A \cap R_{1}=(e)$. En effet, le groupe $A$ opère transitivement sur $R / R_{1}$ et le groupe d'isotropie est égal à $A \cap R_{1}$. Puisque $R$ est simplement connexe et $R_{1}$ est connexe, l'espace quotient $R / R_{1}$ est simplement connexe. Puisque $A \cap R_{1}$ est un sous-groupe discret de $A, A$ est un revêtement de $R / R_{1}$. Il en résulte immédiatement que $A \cap R_{1}=(e)$. Alors le sous espace a de $\mathfrak{r}$ satisfait aux conditions 1 ) et 2 ).

$3^{\circ}$. Supposons que $r_{1}$ n'est pas une sous-algèbre complexe maximale de $r$. Soit $\mathfrak{r}_{2}$ une sous-algèbre complexe maximale de $\mathfrak{r}$ contenant $\mathfrak{r}_{1}$. D'après l'hypothèse de récurrence, $i$ existe des sous-espaces $\mathrm{m}_{l+1}, \ldots, \mathrm{m}_{k}$ de $\mathrm{r}_{2}$ vérifiant les conditions 1 ) et 2 ) pour les groupes $R_{2}$ et $R_{1}$. D'autre part, $\mathfrak{r}_{2}$ étant maximal, d'après ce que l'on a déjà montré, il existe des sous-espaces $m_{1}, \ldots, \mathfrak{m}_{l}$ de $\mathfrak{r}$ tels que $r=m_{1}+\cdots+m_{l}+r_{2}$ et que la condition 2 ) soit vérifiée pour les groupes $R$ et $R_{2}$. Alors les sous-espaces $m_{1}, \ldots, \mathfrak{m}_{k}$ de $\mathfrak{r}$ satisfont aux conditions 1 ) et 2 ) pour les groupes $R$ et $R_{1}$. Le lemme est donc démontré.

Il résulte immédiatement du lemme 3.1 que l'espace quotient $R / R_{1}$ est holomorphiquement homéomorphe à l'espace numérique complexe d'une certaine dimension.

Lemme 3.2. Soit $G$ un groupe de Stein et soit $R$ un sous-groupe de Lie 
fermé, complexe et connexe de $G$. Supposons que $R$ soit résoluble et simplement connexe et que $G / R$ soit une variété de Stein. Soit $K$ un sous-groupe compact maximal de $G$. Alors le groupe $\widetilde{K} \cap R$ est discret ${ }^{2}$.

Soit $R_{1}$ la composante connexe de l'élément neutre du groupe $\widetilde{K} \cap R$. D'après le lemme 3.1, l'espace quotient $R / R_{1}$ est holomorphiquement homéomorphe à l'espace numérique complexe et par suite $R / R_{1}$ est une variété de Stein. L'espace quotient $G / R_{1}$ est un espace fibré holomorphe de base $G / R$ et de fibre $R / R_{1}$. La base $G / R$ et la fibre $R / R_{1}$ étant des variétés de Stein et le groupe structural étant connexe, $G / R_{1}$ est aussi une variété de Stein (voir [7], Théorème 6). La variété $\tilde{K} / R_{1}$ étant une sous-variété complexe fermée de $G / R_{1}$, $\widetilde{K} / R_{1}$ est aussi de Stein (cf. [1]). Le groupe $\tilde{K}$ étant le complexifié du sousgroupe compact maximal $K, R_{1}$ est égal au complexifié d'un sous-groupe compact maximal de $R_{1}$ ([8], Théorème 3 et Remarque 2 au page 216 ). Or, $R_{1}$ étant un sous-groupe du groupe résoluble simplement connexe $R$, tout sous-groupe compact maximal de $R_{1}$ se réduit à $(e)$. Il en résulte que $R_{1}=(e)$ et par conséquent, $\tilde{K} \cap R$ est discret.

Démonstration du thérème 2. D'après le théorème 1 , le groupe $H$ satisfait à la condition $(Q)$. Il existe alors un sous-groupe de Lie fermé, invariant et complexe $N$ de $H$ qui est résoluble, connexe et simplement connexe tel que $H=\widetilde{L} . N, \widetilde{L} \cap N=(e)$. D'autre part, le groupe $G$ est de Stein, car $G$ opère de mainière presque effective sur la variété de Stein $G / H$ (voir [7], Théorèmes 1 et 3). L'espace quotient $G / N$ est un espace fibré principal holomorphe de base $G / H$ et de groupe structural $H / N$. Le groupe $H / N$ est isomorphe au groupe $\tilde{L}$. $H / N$ est donc un groupe de Stein. Alors, d'après [7], Théorème $4, G / N$ est une variètè de Stein. Il résulte alors du lemme 3.2 que le groupe $\widetilde{K} \cap N$ est discret et donc que $\widetilde{\mathfrak{f}} \cap \mathfrak{n}=(0)$. On va montrer que $\widetilde{\mathfrak{f}} \cap \mathfrak{h}=\widetilde{\mathfrak{T}}$. Il est clair que $\tilde{\mathfrak{f}} \cap \mathfrak{h} \supset \tilde{\text { r. }}$ Soit $X \in \tilde{\mathfrak{f}} \cap \mathfrak{h}$. Puisque $\mathfrak{h}=\tilde{\mathfrak{l}}+\mathfrak{n}$ (somme directe), $X$ s'écrit $X=$ $X_{1}+X_{2}, X_{1} \in \mathfrak{l}, X_{2} \in \mathfrak{n}$. Puisque $\widetilde{\mathfrak{I}} \subset \widetilde{\mathfrak{l}} \cap \mathfrak{h}, X-X_{1}$ appartient à $\widetilde{\mathfrak{l}} \cap \mathfrak{h} \cap \mathfrak{n}=\widetilde{\mathfrak{l}} \cap \mathfrak{n}$ et par suite $X=X_{1}$, car $\widetilde{\mathfrak{f}} \cap \mathfrak{n}=(0)$. On a alors $\widetilde{\mathfrak{f}} \cap \mathfrak{h} \subset \tilde{\mathfrak{I}}$ et donc $\widetilde{\mathfrak{f}} \cap \mathfrak{h}=\widetilde{I}$, ce qui

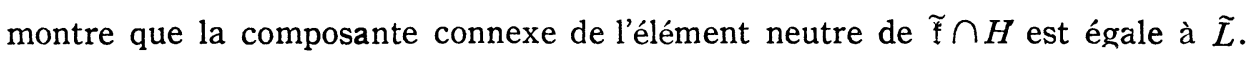
Le théorème 2 est ainsi démontré.

2) Ce lemme a été démontré par A. Morimoto dans le cas où $R$ est abélien. J'ignore si le groupe $\widetilde{K} \cap R$ se réduise à l'élément neutre ou non. 
4. Soit $G$ un groupe de Lie complexe et connexe. On ne supposera pas que $G$ est de Stein. Soit $K$ un sous-groupe compact maximal de $G$. La sousalgèbre complexe $\widetilde{f}$ de $g$ engendrée par la sous-algèbre réelle $\sharp$ n'est pas toujours isomorphe à la complexification $\mathfrak{t}^{c}$ de $₹$. Mais on sait que le groupe $\tilde{K}$ est fermé dans $G[8]$. On va démontrer le théorème suivant qui est un analogue d'un théorème de Mostow [9] (cf. [9], Theorem 2.2).

Thtónème 3. Soit $G$ un groupe de Lie complexe et connexe et soit $K$ un sous-groupe compact maximal de G. Il existe alors des sous-espaces vectoriels complexes $\mathrm{m}_{1}, \ldots, \mathrm{m}_{k}$ de l'algebre de Lie $\mathrm{g}$ satisfaisant aux conditions suivantes;

1) $a=m_{1}+\cdots+m_{k}+\tilde{f}$ (somme directe) et ad.x. $m_{i}=m_{i}$ quel que soit $x \in \widetilde{K}(i=1, \ldots, k)$;

2) l'application exponentielle de $\mathrm{m}_{i}$ dans $G$ est injective $(i=1, \ldots, k)$;

3) tout elément $x$ de $G$ s'écrit de manière unique sous la forme $x=\exp$ $X_{1} \cdots \exp X_{k} . y, y \in \tilde{K}, X_{i} \in \mathrm{m}_{i}(i=1, \ldots, k)$.

Le lemme suivant est facile à démontrer.

Lemme 4.1. Soit $G$ un groupe de Lie complexe et connexe et soit $N$ un sous-groupe de Lie fermé, iuvariant, complexe et connexe de $G$ tel que le groupe quotient $G / N$ soit abelien et simplement connexe. Soit $m$ un sous-espace vectoriel complexe de l'algebre de Lie $\mathfrak{g}$ de $G$ tel que $\mathfrak{g}=\mathfrak{m}+\mathfrak{n}$ (somme directe). Alors l'application exponentielle de $\mathrm{m}$ dans $G$ est injective et tout élément $x$ de $G$ s'écrit de manière unique sous la forme $x=\exp X . y, y \in N, X \in \mathfrak{m}$.

On va démontrer le théorème 3. Soit $Z$ le centre de $G$ et soit $Z_{0}$ la composante connexe de l'élément neutre de $Z$. La représentation adjointe de $G$ induit une représentation linéaire holomorphe fidèle de $G / Z$. D'après la proposition $1.1, G / Z$ satisfait à la condition $(Q)$. Il résulte alors du lemme 2.2 que $G / Z_{0}$ satisfait à la condition $(Q)$. Soit maintenant $L$ le sous-groupe compact maximal de $Z_{0}$. Alors $\widetilde{L}$ est fermé dans $Z_{0}$ et le groupe quotient $Z_{0} / \widetilde{L}$ est simplement connexe. On va montrer que le groupe quotient $G / \widetilde{L}$ satisfait à la condition (Q). En effet, le quotient de $G / \widetilde{L}$ par $Z_{0} / \widetilde{L}$ est isomorphe à $G / Z_{0}$. Il résulte alors du lemme 2.1 que $G / \widetilde{L}$ satisfait à la condition $(Q)$, car $Z_{0} / \widetilde{L}$ est abélien et simplement connexe. Maintenant le sous-groupe compact maximal $K$ de $G$ contient $L$, car $L$ est dans le centre de $G$. Alors $K . \widetilde{L} / \widetilde{L}$ est un sous-groupe compact maximal de $G / \widetilde{L}$ et $\widetilde{K} / \widetilde{L}$ est égal au complexifié de $K \cdot \widetilde{L} / \widetilde{L}$. Puisque 
$G / \widetilde{L}$ satisfait à la condition $(Q)$, il existe un sous-groupe de Lie fermé, invariant, complexe et connexe $N$ de $G$ contenant $\widetilde{L}$ tel que le groupe quotient $N / \widetilde{L}$ soit résoluble et simplement connexe et tel que $G=\widetilde{K} . N, \widetilde{K} \cap N=\widetilde{L}$. Désignons par $D(S)$ le groupe dérivé d'un groupe $S$ et soit $D^{i}(S)=D\left(D^{i-1}(S)\right)$. Soit $N^{\prime}=N / \widetilde{L}$. Le groupe $N^{\prime}$ étant résoluble, il existe un entier $k \geqq 0$ tel que $D^{k}\left(N^{\prime}\right) \neq(e)$ et $D^{k+1}\left(N^{\prime}\right)=(e)$. De plus, $N^{\prime}$ étant simplement connexe, les sous-groupes $D^{i}\left(N^{\prime}\right)$ de $N^{\prime}$ sont tous fermés dans $N^{\prime}$. Il en résulte que les sous-groupes $D^{i}(N) . \widetilde{L}$ de $N$ sont fermés dans $N$ et que $D^{k}(N) \nsubseteq \widetilde{L}$ et $D^{k+1}(N) \subset \widetilde{L}$. Soient $N_{0}=N$, et $N_{i}=D^{i}(N) \cdot \widetilde{L}(i=1, \ldots, k+1)$. Alors $N=N_{0} \supset N_{1} \supset \cdots \supset N_{k} \supset N_{k+1}=\widetilde{L}$ et les sous-groupes $N_{i}$ sont tous invariants et fermés dans $G$. La représentation adjointe de $G$ induit une représentation $\rho_{i}$ de $\tilde{K}$ dans l'espace vectoriel $\mathfrak{n}_{i}(i=1$, $\ldots, k+1)$. On voit facilement que la représentation $\rho_{i}$ est semi-simple $(i=1$, $\ldots, k+1)$. Alors on peut trouver un sous-espace vectoriel complexe $m_{i}$ de $\mathfrak{n}_{i}$ tel que $\mathfrak{n}_{i}=\mathfrak{m}_{i}+\mathfrak{n}_{i+1}$ (somme directe) $(i=1, \ldots, k)$ et que ad. $x \cdot m_{i}=m_{i}$ quel que soit $x \in \tilde{K}$. On a alors $\mathfrak{n}=\mathfrak{m}_{1}+\cdots+\mathfrak{m}_{k}+\mathfrak{n}_{k+1}$ (somme directe). Puisque $\mathfrak{g}=\mathfrak{n}+\widetilde{\mathfrak{f}}, \mathfrak{n} \cap \widetilde{\mathfrak{f}}=\widetilde{\mathfrak{l}}=\mathfrak{n}_{k+1}$, on a $\mathfrak{g}=\mathfrak{m}_{1}+\cdots+\mathfrak{m}_{k}+\widetilde{\mathfrak{f}}$ (somme directe). D'autre part, on voit facilement que $N_{i} / N_{i+1}$ est abélien et simplement connexe. Utilisant le lemme 4.1 plusieurs fois, on voit que l'application exponentielle de $\mathrm{m}_{i}$ dans $N$ est injective $(i=1, \ldots, k)$ et que tout élément $x_{1}$ de $N$ s'écrit de manière unique sous la forme $x_{1}=\exp X_{1} \cdots \exp X_{k} . y_{1}, y_{1} \in \widetilde{L}, X_{i} \in \mathrm{m}_{i}(i=1$, $\ldots, k)$. Soit maintenant $x \in G$. Puisque $G=N . \tilde{K}$, il existe des éléments $x_{1} \in N$ et $x_{2} \in \tilde{K}$ tels que $x=x_{1} \cdot x_{2}$. Soit $x_{1}=\exp X_{1} \cdots \exp X_{k} \cdot y_{1}, y_{1} \in \widetilde{L}, X_{i} \in \mathrm{m}_{i}$ et soit $y=y_{1} \cdot x_{2}$. Alors $y \in \tilde{K}$ et $x$ s'écrit $x=\exp X_{1} \cdots \exp X_{k} \cdot y$. Montrons que cette expression est unique. Soit, en effet, $x=\exp X_{1} \cdots \exp X_{k} \cdot y=\exp X^{\prime}$ $\cdots \exp X_{k}^{\prime} \cdot y^{\prime}, y, y^{\prime} \in \tilde{K}, X_{i}, X_{i}^{\prime} \in \mathrm{m}_{i}(i=1, \ldots, k)$. On a alors $\left(\exp X_{1} \cdots \exp \right.$ $\left.X_{k}\right)^{-1}\left(\exp X_{1}^{\prime} \cdots \exp X_{k}^{\prime}\right)=y \cdot y^{\prime-1} \in N \cap \tilde{K}=\tilde{L}$. Soit $y \cdot y^{\prime^{-1}}=y_{1}$. Alors $y_{1} \in \widetilde{L}$ et $\exp X_{k}^{\prime} \cdots \exp X_{k}^{\prime}=\exp X_{1} \cdots \exp X_{k} \cdot y_{1}$. Alors $X_{i}^{\prime}=X_{i}(i=1, \ldots, k)$ et $y_{1}=e$ et par suite $y=y^{\prime}$. Le théorème 3 est ainsi démontré.

Tirons du théorème 3 quelques conséquences.

1. L'application $\psi$ de $m_{1} \times \cdots \times m_{k} \times \tilde{K}$ sur $G$ définie $\operatorname{par} \psi\left(X_{1}, \ldots, X_{k}, y\right)$ $=\exp X_{1} \cdots \exp X_{k} \cdot y$ est un homéomorphisme holomorphe de la variété complexe $m_{1} \times \cdots \times m_{k} \times \tilde{K}$ sur la variété $G$.

2. Soit $U=\psi\left(m_{1}, m_{2}, \ldots, m_{k}, e\right)$. Alors $x \cdot U \cdot x^{-1}=U$ quel que soit $x \in \widetilde{K}$ : 
Considérons maintenant le cas où $G$ est nilpotent. Alors le sous-groupe compact maximal $K$ de $G$ est contenu dans le centre de $G$. Alors le groupe $\widetilde{K}$ est aussi contenu dans le centre de $G$ et le groupe quotient $G / \widetilde{K}$ est simplement connexe. Soit maintenant $H$ un sous-groupe de Lie, fermé, complexe et connexe de $G$. Alors $H \cdot \widetilde{K}$ est un sous-groupe fermé de $G$, parce que $H \cdot \widetilde{K}$ contient $K$ et que tout sous-groupe de Lie connexe de $G$ contenant un sousgroupe compact maximal est fermé (cf. [8]). Soient $G^{\prime}=G / \widetilde{K}$ et $H^{\prime}=H \cdot \widetilde{K} / \widetilde{K}$. Le groupe $G^{\prime}$ étant nilpotent et simplement connexe, d'après le lemme 3.1 , on peut trouver des sous-espaces vectoriels complexes $m_{1}^{\prime}, \ldots, \mathrm{m}_{h}^{\prime}, \ldots, \mathrm{m}_{k}^{\prime} \mathrm{de}$ dimension complexe 1 de $g^{\prime}$ tels que $g^{\prime}=\mathfrak{m}_{1}^{\prime}+\cdots+\mathfrak{m}_{k}^{\prime}$ (somme directe), $\mathfrak{h}^{\prime}=$ $\mathrm{m}_{1}^{\prime}+\cdots+\mathrm{m}_{h}^{\prime}$ et que tout élément $x^{\prime}$ de $G^{\prime}$ s'écrit de manière unique sous la forme $x^{\prime}=\exp X_{1}^{\prime}, \ldots \exp X_{k}^{\prime}, X_{i} \in \mathrm{m}_{i}^{\prime}(i=1, \ldots, k)$. Soit $\pi^{\prime}$ l'homomorphisme canonique de $g$ sur $g^{\prime}$. Soient $m_{1}, \ldots, m_{k}$ des sous espaces vectoriels complexes de dimension complexe 1 de $\mathfrak{g}$ tels que $\pi^{\prime} \cdot \mathfrak{m}_{i}=\mathfrak{m}_{i}^{\prime}(i=1, \ldots, k)$ et que $\mathfrak{m}_{i} \subset \mathfrak{h}$ $(i=1, \ldots, h)$. Alors $\mathfrak{g}=\mathrm{m}_{1}+\cdots+\mathrm{m}_{k}+\widetilde{\mathfrak{f}}$ (somme directe) $\mathfrak{h}+\widetilde{\mathfrak{f}}=\mathrm{m}_{1}+\cdots$ $m_{h}+\widetilde{f}$ et tout élément $x$ de $G$ s'écrit de manière unique sous la forme $x=e x p$ $X_{1} \cdots \exp X_{k} \cdot y, y \in \tilde{K}, X_{i} \in m_{i}$. De plus, tout élément $x$ de $H \cdot \widetilde{K}$ s'ecrit $x=$ $\exp X_{1} \cdots \exp X_{h} \cdot y, y \in \tilde{K}, X_{i} \in \mathfrak{m}_{i}(i=1, \ldots, h)$. Soit maintenant $x \in H$. Puisque $X_{i} \in \mathfrak{h}(i=1, \ldots, h)$, on a $\exp X_{i} \in H$ et par suite $y$ est un élément de $\tilde{K} \cap H$. On voit donc que tout élément $x$ de $H$ s'ecrit de manière unique sous la forme $x=\exp X_{1} \cdots \exp X_{h} \cdot y, y \in \tilde{K} \cap H, X_{i} \in \mathbb{m}_{i}$. L'application $\varphi$ de $m_{1} \times \cdots \times m_{h} \times(\tilde{K} \cap H)$ sur $H$ définie par $\varphi\left(X_{1}, \ldots, X_{h}, y\right)=\exp X_{1} \cdots \exp$ $X_{h} \cdot y$ est un homéomorphisme et, le groupe $H$ étant connexe, le groupe $\widetilde{K} \cap H$ doit être connexe. Utilisant le fait que $\widetilde{K}$ est contenu dans le centre de $G$, on voit que l'espace quotient $G / H$ est holomorphiquement homéomorphe à la variété complexe $(\tilde{K} / \tilde{K} \cap H) \times m_{h+1} \times \cdots \times \mathfrak{m}_{k}$. Supposons maintenant que $K$ contient un sous-groupe compact maximal $L$ de $H$ et que $\tilde{\imath}=\widetilde{f} \cap \mathfrak{h}$. On a alors $\widetilde{K} \cap H=\widetilde{L}$. Supposons, de plus, que $G$ soit de Stein. On voit alors que le groupe $\widetilde{K} / \widetilde{L}$ est isomorphe au groupe $C^{* m}$ On a ainsi démontré le théorème suivant.

THÉoR ̇̀me 4. Soit $G$ un groupe de Stein nilpotent et soit $H$ un sous-groupe de Lie fermé, complexe et connexe de $G$. Soient $K$ et $L$ des sous-groupes compacts maximaux de $G$ et $H$ respectivement tels que $K \supset L$. Supposons que $H$ satisfasse à la condition $\tilde{f} \cap \mathfrak{h}=\tilde{T}$. Alors l'espace quotient $G / H$ est holomorphi. 
quement homéomorphe à la variete $C^{* m} \times C^{n}$ et le groupe quotient $\tilde{K} / \widetilde{L}$ est isomorphe au groupe $C^{* m}$. En particulier, $G / H$ est une variéte de Stein ${ }^{3)}$.

\section{BIBLIOGRAPHIE}

[1] H. Cartan, Variétés analytiques complexes et cohomologie, Colloque sur les fonctions de plusieurs variables, Bruxelle, 1953, p. 41-45.

[2] C. Chevalley, On the topological structure of solvable groups, Annals of Math., 42, 1941 , p. $669-675$.

[ 3 ] C. Ehresmann, Les prolongements d'une variété différentiable I, Comp. Rendus, Acad. Sci. Paris, 233, 1951, p. 598-600.

[4] H. Grauert, Analytische Faserungen über holomorphe-vollständigen Räumen, Math. Annalen, 135, 1958, p. 263-273.

[ 5 ] K. Iwasawa, On some types of topological groups, Annals of Math., 50, 1949, p. 507558.

[6] G. Hochschild and G. D. Mostow, Representations and representative functions of Lie groups, III, Annals of Math., 70, 1959, p. 85-100.

[7] Y. Matsushima et A. Morimoto, Sur certains espaces fibrés holomorphes sur une variété de Stein, Bull. Soc. math. France, 88, 1960, p. 137-155.

[8] Y. Matsuhsima, Espaces homogènes de Stein des groupes de Lie complexes, Nagoya Math. Journ., 16, 1960, p. 205-218.

[9] G. D. Mostow, On covariant fiberings of Klein spaces, Amer. Journ. Math., 77, 1955, p. $247-278$.

Institut de Mathématiques

Université d'Osaka

3) Ce théorème a été démontré aussi par $M$. Ise par une méthode analogue. 